\title{
Identification of SOFT syndrome caused by a pathogenic homozygous splicing variant of POC1A: a case report
}

\author{
Guoqiang $\mathrm{Li}^{1 \dagger}$, Guoying Chang ${ }^{2 \dagger}$, Chen Wang ${ }^{1}$, Tingting $\mathrm{Yu}^{1}$, Niu Li ${ }^{1}$, Xiaodong Huang ${ }^{2}$, Xiumin Wang ${ }^{2}$, \\ Jian Wang ${ }^{1}$, Jiwen Wang ${ }^{3^{*}}$ and Ruen Yao ${ }^{1 *}$ (D)
}

\begin{abstract}
Background: Pathogenic variants in POC1A led to SOFT syndrome and variant POC1A-related (VPOC1A) syndrome. SOFT syndrome is a rare primordial dwarfism condition characterized by short stature, onychodysplasia, facial dysmorphism and hypotrichosis. The main clinical differences between SOFT and VPOC1A syndrome include dyslipidemia with insulin resistance and acanthosis nigricans. To our knowledge, this is the first report of a SOFT syndrome patient diagnosed with a homozygous splicing variant, which could help to extend our understanding of the genotypic and phenotypic information of the disease.
\end{abstract}

Case presentation: We reported a seven-year-old boy with SOFT syndrome. The patient presented symmetrical short stature and facial features, including prominent forehead, inverted triangular face, epicanthal fold, small teeth and enlarged ears. Laboratory tests displayed mild insulin resistance. Whole-exome sequencing (WES) led to the identification of a homozygous splicing variant (c.981+1G>A) in POC1A gene of the patient, which was inherited from his heterozygous parents confirmed by Sanger sequencing. Further transcriptional experiments of the splicing variant revealed aberrant percentage of exon 9 skipping transcripts.

Conclusions: This is the firstly reported case of a SOFT syndrome patient with a novel homozygous splicing variant and detailed delineation of the aberrant transcript in proband and carrier of the variant in Chinese. Our study enriched mutational spectrum of POC1A which could help in further genetic diagnosis and counselling of SOFT syndrome patients.

Keywords: SOFT syndrome, POC1A, Splicing variant, Short stature, Case report

\section{Background}

SOFT syndrome (OMIM 614813) is a rare genetic disorder, which is the abbreviation of Short stature,

\footnotetext{
*Correspondence: wangjiwen@scmc.com.cn; yaoruen@126.com

${ }^{\dagger}$ Guogiang Li and Guoying Chang contributed equally

1 Department of Medical Genetics and Molecular Diagnostic Laboratory, Shanghai Children's Medical Center, School of Medicine, Shanghai Jiao

Tong University, Shanghai 200127, People's Republic of China

${ }^{3}$ Department of Neurology, Shanghai Children's Medical Center, School

of Medicine, Shanghai Jiao Tong University, Shanghai 200127, People's

Republic of China

Full list of author information is available at the end of the article
}

Onychodysplasia, Facial dysmorphism and hypoTrichosis, initially termed by Sarig et al. in 2012 [1]. SOFT syndrome is caused by POC1A gene mutation and is inherited in an autosomal recessive manner. There are three reviewed mRNA isoforms for POC1A gene, one of which lacks exon 10. POC1A gene has pleiotropic effects, because truncating variants locating in exon 10 could cause variant POC1A-related (vPOC1A) syndrome, whose main clinical features included an extreme dyslipidemia with insulin resistance, acanthosis nigricans and short stature. To date, only 15 families with molecularly 


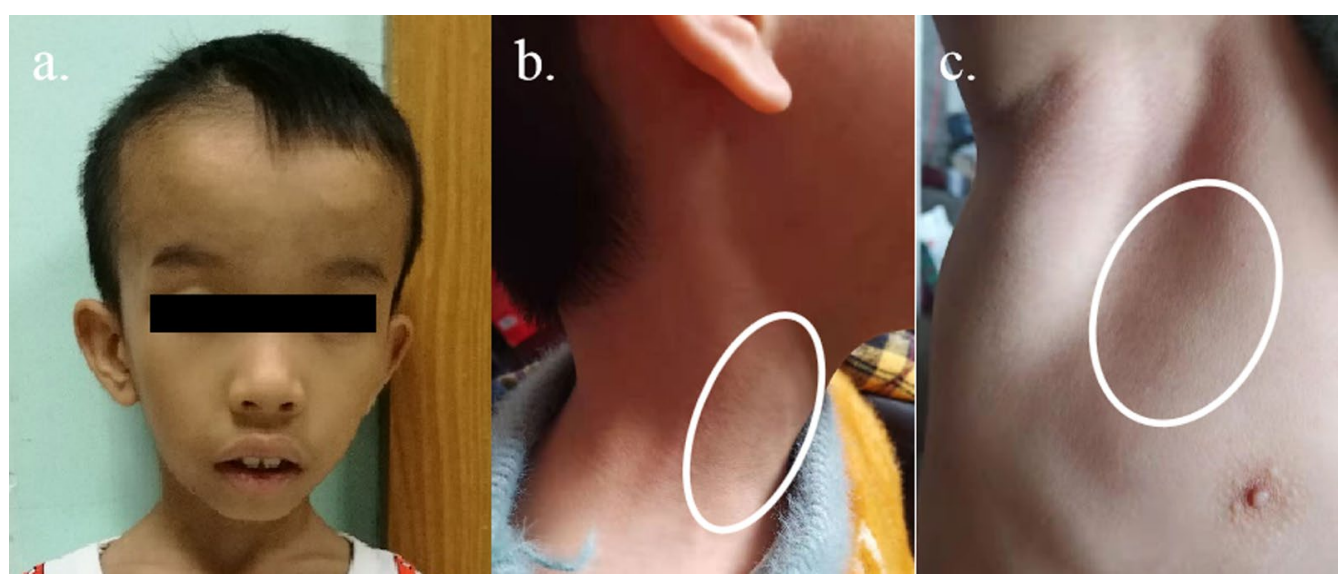

Fig. 1 The phenotype of the patient. a Facial dysmorphism including prominent forehead, inverted triangular face, epicanthal fold, small teeth and enlarged ears. b, c Mild acanthosis nigricans inneck and axillary fossa, the acanthosis nigricans was indicated by a circle

confirmed variants in $P O C 1 A$ gene have been reported [1-12]. POC1A gene encodes the POC1 centriolar protein $\mathrm{A}$, which is one of the two proteome of centriole 1 (POC1) proteins in humans. The encoded protein consists of an N-terminal WD40 domain and a C-terminal coiled coil domain, named the Poc1 motif, and is involved in centriole duplication and length control [13]. Here, we report the first Chinese patient of SOFT syndrome harboring a homozygous splicing variant of $P O C 1 A$ gene.

\section{Case presentation}

The proband was a seven-year-old boy. He was referred to the endocrinology clinic of Shanghai Children's Medical Center (SCMC) for the chief complaint of short stature and facial dysmorphism. He was the third child of non-consanguineous parents of the Han nationality. His parents had an induced labour for absence of one kidney for fetus. His parents were physically healthy without relevant family history. The height of his father was $172 \mathrm{~cm}$ and mother $160 \mathrm{~cm}$ (Fig. 2a).

The child was delivered by normal labor at full term gestation. During pregnancy, prenatal examination indicated that he had short legs and arms. His birth weight was $2300 \mathrm{~g}$ [ $-2.6 \mathrm{SD}]$ and length at birth was $46.8 \mathrm{~cm}$ [ $-2 \mathrm{SD}$ ]. When ten months old, he was taken to the local hospital due to growth retardation and small appetite, and a diagnosis of thyroid dysgenesis was made. Then, he took the sodium levothyroxine for 1 year, but stopped taking it because of no efficacy. Mild speech delay and motor developmental delay were described by his parents. The height he gained was less than $1 \mathrm{~cm}$ in the past year. His intelligence was normal. Upon physical a.

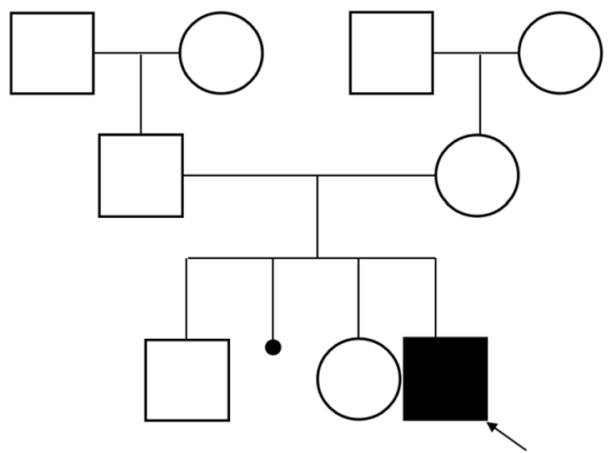

b.

c. $981+1 \mathrm{G}>\mathrm{A}$

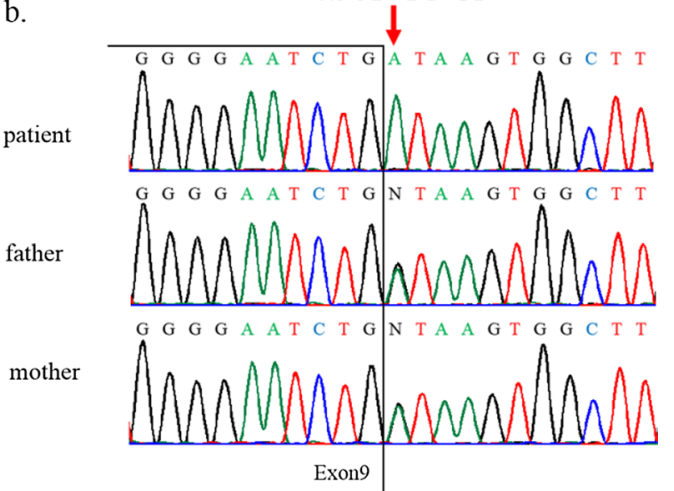

Fig. 2 a The pedigree of the patient. The proband was indicated by an arrow. $\mathbf{b}$ Identification of a splicing variant in the POC1A gene. Sequences show a homozygous splicing variant (c.981+1G>A in intron 9) in the patient. The patient's father and mother were both carriers. Red arrows, mutant bases 
examination, his height was $90 \mathrm{~cm}(-6.3 \mathrm{SD})$, weight $10.3 \mathrm{~kg}(-3.9 \mathrm{SD})$, and his head circumference was $48 \mathrm{~cm}$ $(<-2 \mathrm{SD})$. He presented facial dysmorphism including prominent forehead, inverted triangular face, epicanthal fold, small teeth and enlarged ears (Fig. 1a). And mild acanthosis nigricans occurred in his neck and axillary fossa (Fig. 1b, c). His vision and hearing was normal.

The laboratory results showed mild insulin resistance. Because of his compliance, we examined his blood glucose and insulin via a simple oral glucose tolerance test. The results showed that the fasting glucose value was $5.05 \mathrm{mmol} / \mathrm{L}$ (reference $4.1-5.9 \mathrm{mmol} / \mathrm{L}$ ), and the fasting insulin value was $90.28 \mathrm{pmol} / \mathrm{L}$ (reference $12-84 \mathrm{pmol} / \mathrm{L}$ ). And two hours after having a meal, the level of glucose was $5.29 \mathrm{mmol} / \mathrm{L}$ (reference $4.1-5.9 \mathrm{mmol} / \mathrm{L}$ ), and the insulin was $433.99 \mathrm{pmol} / \mathrm{L}$ (reference $12-84 \mathrm{pmol} / \mathrm{L}$ ). The level of HbA1c was 5.6\% (reference 4.0-6.0\%). The level of insulin like growth factor 1 (IGF1) and insulin like growth factor binding protein 3 (IGFBP3) was $190.0 \mathrm{ng} /$ $\mathrm{ml}(-0.5 \mathrm{SD})$ and $3.84 \mathrm{ug} / \mathrm{ml}(-1.5 \mathrm{SD})$, respectively. The Vitamin D was $20.0 \mathrm{ng} / \mathrm{ml}$ (reference $>30.0 \mathrm{ng} / \mathrm{ml}$ ). Laboratory investigations showed levels within the reference ranges including free triiodothyronine (FT3), free tetraiodothyronine (FT4) and thyroid stimulating hormone (TSH). Moreover, the liver and kidney function, blood cholesterol and blood triglycerides were also normal. The echocardiography was normal but the electrocardiograph indicated a prolongation of the QT interval when tachycardia. The X-ray indicated a bone age of 3.5-year-old. And the ultrasound of heart and abdomen was normal.

In order to detect disease-causing mutations, genomic DNA was extracted from peripheral blood samples of patient and his parents using the Gentra Puregene Blood Kit (Qiagen, Hilden, Germany) according to the manufacturer's protocol. Whole exome capture was performed with Agilent SureSelect V6 enrichment capture kit (Agilent Technologies, Inc., Woburn, MA, U.S.) according to the manufacturer's instructions. The captured library was then sequenced on an Illumina HiSeq 2500 System (Illumina, Inc., San Diego, CA, U.S.). Original sequencing data were assessed using FastQC (version 0.11.2) for quality control. The Burrows Wheeler Alignment tool (BWA) v0.2.10 was employed for sequencing data alignment to the Human Reference Genome (NCBI build 37, hg 19).
Single nucleotide variants and small indels were identified by GATK. All variants were saved in VCF format and uploaded to the Ingenuity Variant Analysis (Ingenuity Systems, Redwood City, CA, USA) and TGex (Translational Genomics Expert) platform for biological analysis and interpretation. Variants detected by next-generation sequencing were confirmed by Sanger sequencing in the patient and his parents. The primers designed for exon 9 were as follows: forward $5^{\prime}$-CTCAGAACTTTGGGC ATGGC- $3^{\prime}$ and reverse $5^{\prime}$-CTAAACCTCCTCCCTCAC CG-3'. Transcriptional experiments were performed to evaluate the splicing effects of the variant. The cDNA products were amplified with primer P1, 5'-CTCGTA TTGTGAGCATGGCG-3', which was located in the exon6; and primer P2, 5'-GATTCCTGCTCCCCTGAT CA-3', which was located in exon11. The polymerase chain reaction (PCR) products were further cloned into the pMD $19 \mathrm{~T}$ vector (Takara Biotechnology [Dalian] Co., Ltd.). The polymerase chain reaction was carried out in a $\mathrm{C}_{1000}{ }^{\mathrm{TM}}$ Thermal Cycler PCR instrument (Bio-Rad Laboratories). The resulting DNA was sequenced using an ABI3730XL sequencer (Thermo Fisher Scientific) with the forward and reverse primers. The sequence data were analyzed using Mutation Surveyor ${ }^{\circledR}$ software version 4.0.4 (SoftGenetics, LLC).

Whole-exome sequencing revealed a novel homozygous splicing variant, c. $981+1 \mathrm{G}>\mathrm{A}$, in intron 9 of the POC1A gene (NM_015426.5) in the patient. Sanger sequencing confirmed the variant and heterozygous status in his father and mother (Fig. 2b). The variant detected in the family was not included in control population database (gnomAD) and our local control cohorts database.

Reverse transcription polymerase chain reaction (RTPCR) performed on RNA extracted from the patient, parents and control subject, revealed the existence of aberrant transcripts percentage derived from the splicing variant. Besides the shorter isoform of POC1A lacking exon 10 which had been proved to existed in different tissues, a significant altered percentage of mutant isoform lacking exon 9 and both exon $9 \& 10$ had been discovered between the proband, the parents and control sample (Table 1, Additional file 1: Data 1). The exon 9 and exon 9\&10 skipping transcripts both leading to short protein

Table 1 isoforms of POC1A transcript detected in control sample and the pedigree samples

\begin{tabular}{lcccc}
\hline & Full-length isoform & 10-isoform & 9-isoform & 9- and 10- isoform \\
\hline Control & $62 / 74(83.78 \%)$ & $12 / 74(16.22 \%)$ & $0 / 74(0.00 \%)$ & $0 / 74(0.00 \%)$ \\
Carrier (father) & $29 / 76(38.16 \%)$ & $5 / 76(6.58 \%)$ & $34 / 76(44.74 \%)$ & $8 / 76(10.53 \%)$ \\
Carrier (mother) & $27 / 76(35.53 \%)$ & $3 / 76(3.95 \%)$ & $37 / 76(48.68 \%)$ & $9 / 76(11.84 \%)$ \\
Proband & $0 / 80(0.00 \%)$ & $0 / 80(0.00 \%)$ & $69 / 80(86.25 \%)$ & $11 / 80(13.75 \%)$ \\
\hline
\end{tabular}


product with in-frame deletion of 33 and 81 amino acids respectively.

\section{Discussion and conclusions}

SOFT syndrome is characterized by short stature, facial dysmorphism with hypotrichosis and facial dysmorphism. Pathogenic variants in POC1A gene can lead to SOFT syndrome. The growth retardation can be detected on prenatal ultrasound $[1,2]$. Relative macrocephaly is present in childhood but the head circumference becomes smaller with advancing age $[1,2]$. Skeletal anomalies like clinodactyly, brachydactyly and hypoplastic distal phalanges have been reported. Recognizable facial dysmorphism includes elongated triangular face, prominent nose and abnormal ears [6]. In addition, affected patients have a high-pitched voice [9]. The patients usually have normal psychomotor development and intelligence.

$P O C 1 A$ encodes the POC1 centriolar protein $\mathrm{A}$, which plays a role in centrosome-mediated cell mitosis control via mitotic spindle organization and cilia formation. Formation of supernumerary centrosomes and multipolar spindle were observed in fibroblast cells derived from the affected individuals $[1,3]$. Therefore, SOFT syndrome could be classified as a type of ciliopathy. To our best knowledge, only 13 variants of POC1A gene have been reported until now, including 7 missense mutations, 2 nonsense mutations and 4 frameshift mutations [1-12] (Fig. 3).

Giorgio E et al. suggested that the $P O C 1 A$ gene was a gene with pleiotropic effects, and variants affecting exon 10 only could cause variant POC1A-related (vPOC1A) syndrome instead of typical SOFT syndrome, whose main clinical features included an extreme dyslipidemia with insulin resistance and acanthosis nigricans, because variants restricted to exon 10 of the POC1A gene affected only two of the three mRNA isoforms, leaving a functional isoform [7]. To date, three vPOC1A syndrome individuals had been reported $[4,7,12]$. The splicing variant detected in our patient has been proven to cause exon 9 skipping which are necessary in all isoforms and affect WD40 domain integrity. The WD40 domain is necessary to localize the protein to centrioles, and defective WD40 domain will inhibit the proper protein localization. The parents, heterozygous carriers of the variant, were also significantly affected, producing only about $40 \%$ normal transcripts of POC1A. These findings provided evidences for possible pathogenic mechanism of dosage effect of variants in $P O C 1 A$ gene.

In current study, our patient's phenotypes corresponded with major characteristics of SOFT syndrome, and present with an extra diagnosis of thyroid dysgenesis and mild insulin resistance, which was different from previous reported cases with SOFT and vPOC1A syndrome. We supposed that the reason was that he was at prepubertal age, when severe insulin resistance was yet to be clinically expressed [7]. Further evaluation on his metabolism is needed in follow-up visit from puberty onwards to explore the existence of severe insulin resistance, which is a significant feature for $\mathrm{vPOC} 1 \mathrm{~A}$ syndrome.

In summary, we reported the first patient with SOFT syndrome harboring a homozygous splicing variant of POC1A which leads to aberrant transcripts lacking exon 9 which possibly affected WD40 domain structure of the protein. The patient presented with typical SOFT syndrome features including primordial dwarfism, characteristic facial dysmorphism and delayed bone age, as well as mild insulin resistance. Identification

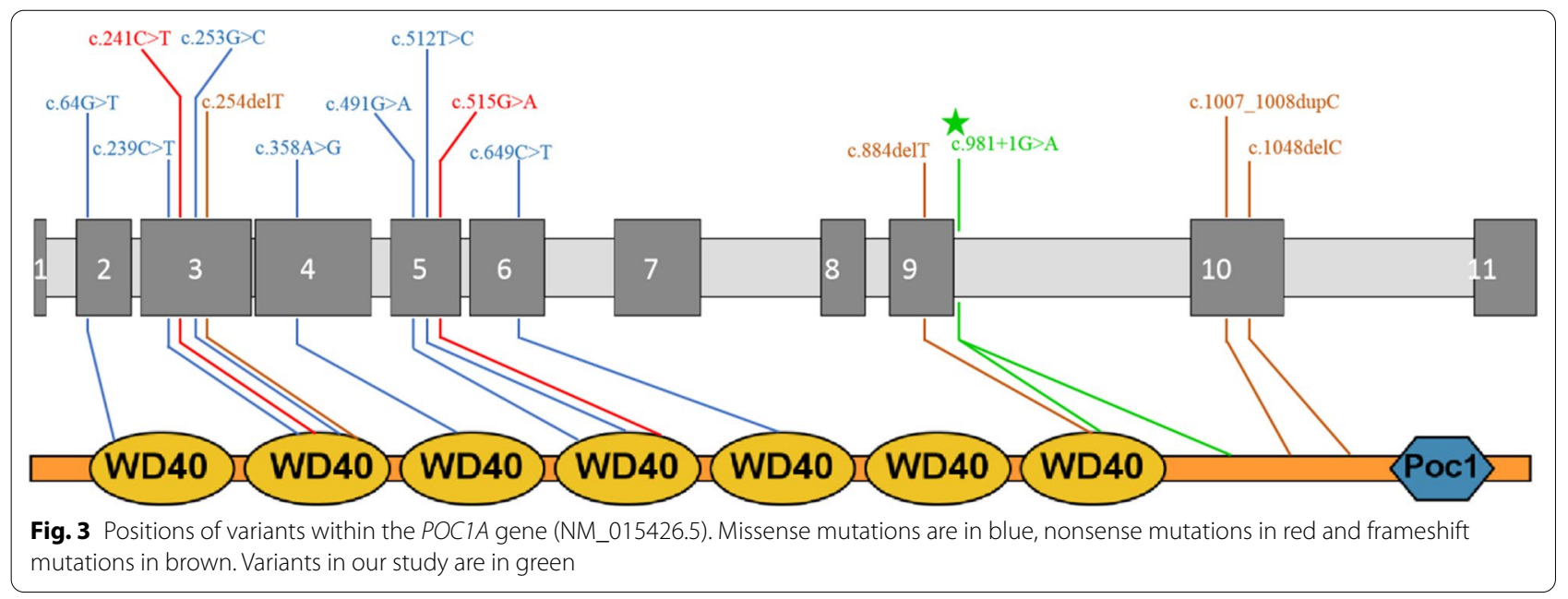


of novel types of POC1A mutation in patients may provide appropriate management and correct genetic counseling to those patients and their families.

\section{Abbreviations}

WES: Whole-exome sequencing; PCR: Polymerase chain reaction; RT-PCR: Reverse transcription polymerase chain reaction; SD:: Standard deviation.

\section{Supplementary Information}

The online version contains supplementary material available at https://doi. org/10.1186/s12920-021-01055-1.

Additional file 1. Data 1 contains detail presentation of normal and aberrant transcripts of the proband, his parents and normal control.

\section{Acknowledgements}

We would like to express our sincere gratitude to our patient and his family for their cooperation.

\section{Authors' contributions}

REY and JWW designed and organized the study. XDH, XMW and GYC sampled the family members and acquired the clinical data. GQL and CW carried out the molecular genetic testing. JW and NL analyzed and interpreted the genetic testing and clinical data. GQL, GYC and TTY wrote the manuscript, which was then revised by XDH and XMW. Finally, REY edited it before submission. All authors read and approved the final manuscript.

\section{Funding}

This study was financially supported by the National Natural Science Fund of China(82001371 and 81900722). The fund was mainly used for transcriptional experiments. Dr. Ruen Yao and Dr. Guoying Chang were in charge of the projects respectively.

\section{Availability of data and materials}

The novel variant revealed during the study has been submitted to ClinVar repository with the accession number SCV001769404 (https://www.ncbi.nlm. nih.gov/clinvar/variation/1192285/). The datasets generated during the current study are available in the NCBI Sequence Read Archive Database (Accession Number: PRJNA753550) (https://www.ncbi.nlm.nih.gov/bioproject/PRJNA 753550). The relevant datasets links used in this study were as follows: Human Reference Genome (GRCh37/hg19) (http://hgdownload.soe.ucsc.edu/golde nPath/hg19/bigZips/hg19.fa.gz), gnomAD (https://gnomad.broadinstitute. org), 1000 Genomes project (http://www.1000genomes.org/), dbSNP (http:// www.ncbi.nlm.nih.gov/snp), ClinVar (https://www.ncbi.nlm.nih.gov/clinvar/), and OMIM (http://omim.org).

\section{Declarations}

\section{Ethics approval and consent to participate}

A seven-year-old boy with main complaint of short stature and facial dysmorphism was enrolled in our studies. This study was approved by the Committee on Ethics of the Shanghai Children's Medical Center (SCMCIRB-K2016013) and was performed in accordance with the Declaration of Helsinki. Written informed consent was obtained from the proband's parents and the control subject to participate in our study.

\section{Consent for publication}

Written informed consent for publication of identifying images and other personal or clinical details was obtained from both of the proband's father and mother. Written informed consent for publication of identifying images or other personal or clinical details was obtained from the control subject.

\section{Competing interests}

The authors declare that they have no competing interests.

\section{Author details}

${ }^{1}$ Department of Medical Genetics and Molecular Diagnostic Laboratory, Shanghai Children's Medical Center, School of Medicine, Shanghai Jiao Tong University, Shanghai 200127, People's Republic of China. ${ }^{2}$ Department of Endocrine and Metabolism, Shanghai Children's Medical Center, School of Medicine, Shanghai Jiao Tong University, Shanghai 200127, People's Republic of China. ${ }^{3}$ Department of Neurology, Shanghai Children's Medical Center, School of Medicine, Shanghai Jiao Tong University, Shanghai 200127, People's Republic of China.

Received: 14 May 2021 Accepted: 12 August 2021

Published online: 21 August 2021

\section{References}

1. Sarig O, Nahum S, Rapaport D, et al. Short stature, onychodysplasia, facial dysmorphism, and hypotrichosis syndrome is caused by a POC1 A mutation. Am J Hum Genet. 2012;91(2):337-42.

2. Shaheen R, Faqeih E, Shamseldin HE, et al. POC1A truncation mutation causes a ciliopathy in humans characterized by primordial dwarfism. Am J Hum Genet. 2012;91(2):330-6.

3. Koparir A, Karatas OF, Yuceturk B, et al. Novel POC1A mutation in primordial dwarfism reveals new insights for centriole biogenesis. Hum $\mathrm{Mol}$ Genet. 2015;24(19):5378-87.

4. Chen JH, Segni M, Payne F, et al. Truncation of POC1A associated with short stature and extreme insulin resistance. J Mol Endocrinol. 2015:55(2):147-58.

5. Ko JM, Jung S, Seo J, et al. SOFT syndrome caused by compound heterozygous mutations of POC1A and its skeletal manifestation. J Hum Genet. 2016;61(6):561-4.

6. Barraza-García J, Iván Rivera-Pedroza C, Salamanca L, et al. Two novel POC1A mutations in the primordial dwarfism, SOFT syndrome: clinical homogeneity but also unreported malformations. Am J Med Genet A. 2016;170A(1):210-6.

7. Giorgio E, Rubino E, Bruselles A, et al. A syndromic extreme insulin resistance caused by biallelic POC1A mutations in exon 10. Eur J Endocrinol. 2017;177(5):K21-7.

8. Bourchany A, Thauvin-Robinet C, Lehalle D, et al. Reducing diagnostic turnaround times of exome sequencing for families requiring timely diagnoses. Eur J Med Genet. 2017;60(11):595-604.

9. Mostofizadeh N, Gheidarloo M, Hashemipour M, Dehkordi EH. SOFT syndrome: the first case in Iran. Adv Biomed Res. 2018;7:128.

10. Saida K, Silva S, Solar B, et al. SOFT syndrome in a patient from Chile. Am J Med Genet A. 2019;179(3):338-40.

11. Al-Kindi A, Al-Shehhi M, Westenberger A, et al. A novel POC1A variant in an alternatively spliced exon causes classic SOFT syndrome: clinical presentation of seven patients. J Hum Genet. 2020;65(2):193-7.

12. Majore S, Agolini E, Micale L, et al. Clinical presentation and molecular characterization of a novel patient with variant POC1A-related syndrome. Clin Genet. 2021;99(4):540-6.

13. Keller LC, Geimer S, Romijn E, et al. Molecular architecture of the centriole proteome: the conserved WD40 domain protein POC1 is required for centriole duplication and length control. Mol Biol Cell. 2009;20(4):1150-66.

\section{Publisher's Note}

Springer Nature remains neutral with regard to jurisdictional claims in published maps and institutional affiliations. 